\title{
The Design, Development and Construction of an Adjustable Lower Extremity
}

\author{
Albert E. Yousif $^{1}$, Ahmed Ali Sadiq $^{2}$ \\ ${ }^{1}$ (Albert E. Yousif is a professor at Medical Engineering Department, Alnahrain University. Baghdad, Iraq. \\ ${ }^{2}$ (Ahmed Ali Sadiq is a graduate student at Medical Engineering Department, Alnahrain University. Baghdad,
}

\begin{abstract}
A simple, low cost yet effective lower limb prosthesis is presented for use in our country and the third world. The purpose of the limb is to enable mobility to amputees and help them in standing, kneeling and maintaining balance. It includes the design and development of knee joint, adjustable shank, ankle joint and foot. The length of shank can be adjusted easily to suit many types of amputee levels (above knee and below knee) and can withstand a good range of patient heights and weights.
\end{abstract}

Keywords - Prosthesis, lower limb, adjustable shank, design.

\section{INTRODUCTION}

The lower limb prosthesis is a device that substitutes a part of a limb missing either due to amputation or a congenital defect. The prosthesis is assembled using off-the-shelf components and a custom-made socket for its attachment to the residuum [1]. For amputees, multiple replacement limbs and repairs are necessary over a lifetime. Children between the ages of four and sixteen grow at an average rate of $2 \mathrm{~cm}$ annually. For example, if a child becomes limb deficient at the age of 10, he will need approximately 25 limbs throughout the course of his or her lifetime. However, if persons become limb deficient while they are adults, they typically will go through about 15-20 limbs during their lifetime (Prosthetics Outreach Foundation, 2005). A prosthetic replacement is needed typically every 6-12 months for children, and every 3-5 years for adults [2].

For children amputees, although many prosthetic principles used in treating adults apply to the treatment of children as well, the child with a lower-limb deficiency presents the prosthetic designer with a unique range of considerations, both practical and philosophical. Most techniques used with adult amputees must be downsized, sequenced in degree of complexity, modified or completely altered to match the everchanging needs of children.

At the growing age in children there is a need of change of prosthesis after 6 months or even earlier to keep the force distribution equal in both limbs and to avoid limb length discrepancy [3].

The aim of the present study is to design and develop lower limb prosthesis including: foot, ankle joint, adjustable length shank and knee joint. This artificial limb can be used by adult amputees and also by children amputees during a significant period of time without having to change the limb during their growth.

\section{ANALYSIS OF THE LOWER LIMB}

A two-dimensional model of the prosthesis was considered. The assumptions of this model were:

1- Moments and movements were taken in the sagittal plane (which divides the body vertically into right and left halves) plane only; where the largest motions take place, which means that the joints have single degree-of-freedom motion.

2- Joints friction forces are neglected.

The models consist of three rigid parts: thigh, shank and foot, connected by two hinge joints represent the knee and ankle. See Fig. (1). 


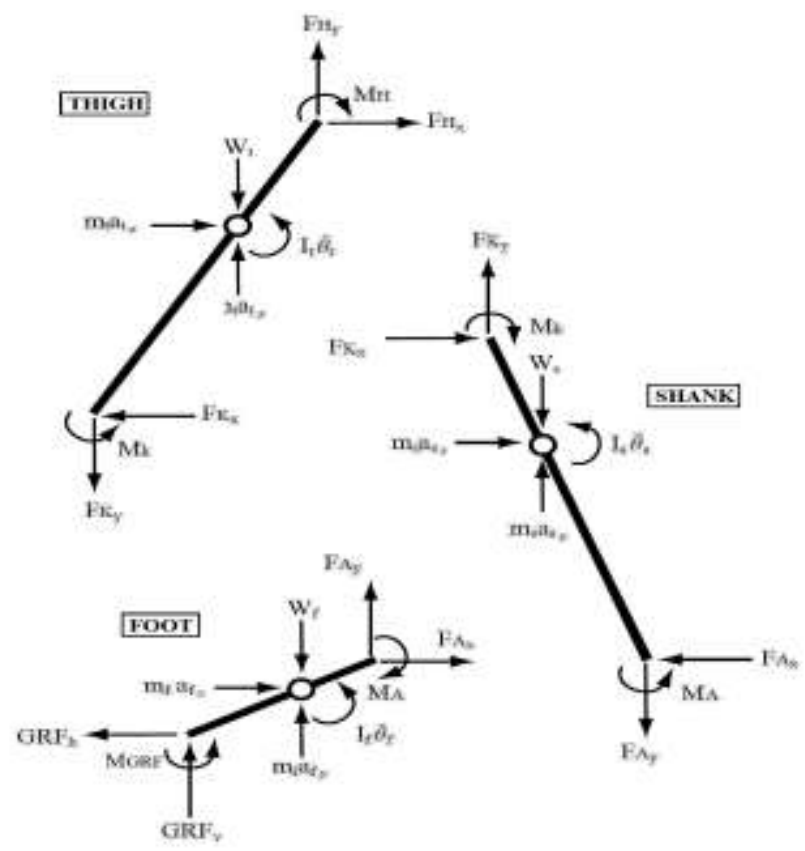

Fig. (1) Free body diagrams of the human lower extremity segments during gait cycle [4].

Fig. (1) shows the free body diagrams of the lower extremity segments (thigh, shank, and foot) that demonstrate each segment forces isolated from the other segment forces. The link-segment model is broken down at the joints into segments, which are treated separately as rigid bodies, creating a free-body diagram.

Bodies in motion are considered to be in a state of dynamic equilibrium with all acting forces resulting in equal and oppositely directed inertia forces; this general concept is known as D'Almbert's principle, this principle depends on Newton's second law $(\mathrm{F}=\mathrm{ma})$, which incorporates factors known as inertia vectors; describe the conditions of dynamic equilibrium. The equations of dynamic equilibrium in two dimensions may be stated as follows:

$\Sigma F_{x}-m a_{x}=0$
$\Sigma F_{y}-m a_{y}=0$
$\Sigma T_{G}-I \alpha=0$

The sums of the horizontal and vertical forces acting on a body are $\Sigma \mathrm{F}_{\mathrm{x}}$ and $\Sigma \mathrm{F}_{\mathrm{y}} ; \mathrm{ma}_{\mathrm{x}}$ and $\mathrm{ma}_{\mathrm{y}}$ are the products of body's mass and the horizontal and vertical accelerations of the body's center of mass; $\Sigma \mathrm{T}_{\mathrm{G}}$ is the sum of torques about the body's center of mass, $\mathrm{I}_{\alpha}$ is the product of body's moment of inertia about the center of mass and the body's angular acceleration [5].

Forces and moments at the Thigh

$\Sigma F_{t x}-m_{t} a_{t}=0$

$F_{H x}-F_{k x}=m_{t} a_{t x}$

$\Sigma F_{t y}-m_{t} a_{t y}=0$

$F_{H y}-F_{K y}-W_{t}=m_{t} a_{t y}$

$I_{t} \ddot{\theta}_{t}+M_{K}-M_{H}=0$

$M_{K}-M_{H}=-I_{t} \ddot{\theta}_{t}$

Forces and moments at the Shank

$\Sigma F_{s x}-m_{s} a_{s x}=0$

$F_{K x}-F_{A x}=m_{s} a_{s x}$

$\Sigma F_{s y}-m_{s} a_{s y}=0$

$W_{s}-F_{A y}+F_{K y}=m_{s} a_{s y}$

$\Sigma T_{s}-I_{s} \ddot{\theta}_{s}=0$

$I_{s} \ddot{\theta}_{S}+M_{A}-M_{K}=0$

$M_{A}-M_{K}=-I_{s} \ddot{\theta}_{s}$

Forces and moments at the Foot

$\Sigma F_{f x}-m_{x f} a_{f x}=0$ 
$G R F_{h}-F_{A x}=m_{f} a_{f x}$

$\Sigma F_{f y}-m_{f} a_{f y}=0$

$G R F_{v}-W_{f}-F_{A y}=m_{f} a_{f y}$

$\Sigma T_{f}-I_{f} \ddot{\theta}_{f}=0$

$I_{f} \ddot{\theta}_{f}+M_{G R F}-M_{A}=0$

$M_{G R F}-M_{A}=-I_{f} \ddot{\theta}_{f}$

\section{DESIGN OF THE ADJUSTABLE LIMB}

The endoskeletal sytem for the design of artificial limb was used in this section because it is more reliable, widely used and each component can be replaced easily or attached to other types of components without changing the whole limb device. The limb components drawing were obtained using AutoCAD 2010 and ANSYS v.12.1 Design Modeler. These components include: knee joint, adjustable shank, ankle joint and foot. The major focus in the design will be on the adjustable shank. The components of the limb are designed to fit a broad demographic subject's height range and withstand different levels of amputees mass depending on the types of materials used to manufacture it. Each part of the limb is detachable and can be replaced or used with other compatible designs.

The knee joint is designed as a hinge joint that has one degree of freedom since as it is considered in many references in literature. The maximum flexion angle is approximately 125 degree. It has a manual locking system. The lock can be released and a compression spring with variable stiffness (depends on the patient's weight) can be attached on the back side to enable the amputee to slightly flex the knee during walking and add more symmetry, comfort to the gait. The upper end is pyramidal in shape and can be attached to any compatible pyramidal adapter or socket adapter. The lower end is an open ended cylinder with locking screw to be attached to the shank, see fig. (2).

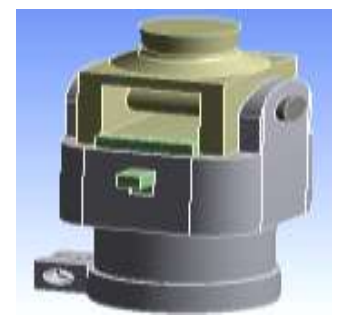

Fig. (2) The Knee Joint.

Stainless steel is a good material option to be used to manufacture the knee joint. It has a high yield stress, good wear and tear properties, corrosion resistance and it is widely available. The only concern is the relatively high weight of the manufactured knee that may be not convenient for subjects with weak muscles or have very short stump length.

The shank has upper and lower parts that can slide inside each other to reach the desired length of the shank then locked with a screw and can be adjusted very easily. The length of the shank can be varied from 275 $\mathrm{mm}$ to $400 \mathrm{~mm}$ with a range of $125 \mathrm{~mm}$. Each part is $225 \mathrm{~mm}$ in length and this can be increased for longer patients or decreased for children. See fig. (3).

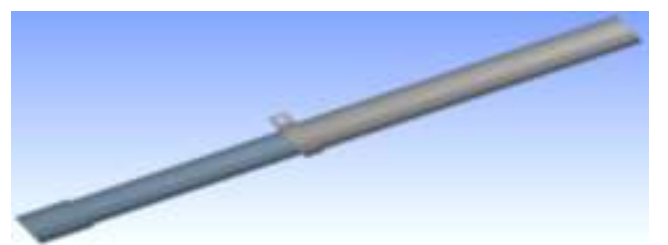

Fig. (3) The Adjustable Shank.

To determine the best length of the two parts, a computer program was used to draw these parts and how they slide inside each other. The approach is illustrated in fig. (4). 


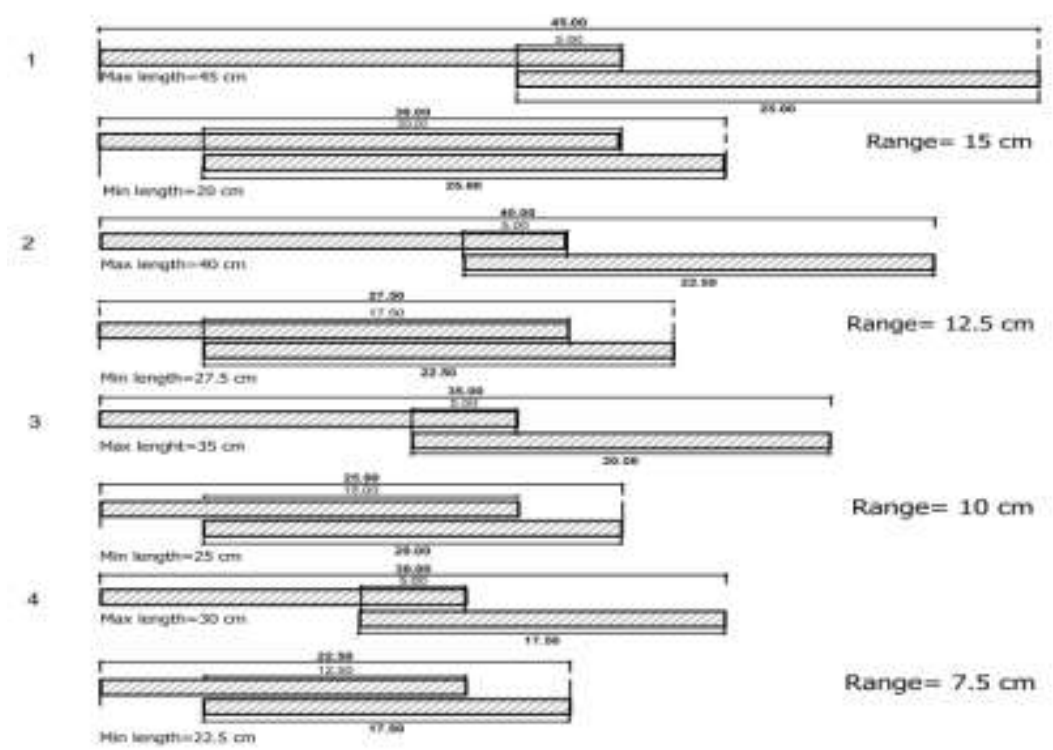

Fig. (4) Drawing of the parts of the adjustable shank.

Four cases were examined and the maximum lengths, minimum lengths and ranges were calculated. Table (1) presents these numbers.

Table (1) Determining the optimal length of the parts of the adjustable shank

\begin{tabular}{|c|c|c|c|c|}
\hline & Part length $(\mathrm{cm})$ & Max length $(\mathrm{cm})$ & Min length $(\mathrm{cm})$ & Range $(\mathrm{cm})$ \\
\hline 1 & 25 & 45 & 30 & 15 \\
\hline 2 & 22.5 & 40 & 27.5 & 12.5 \\
\hline 3 & 20 & 35 & 25 & 10 \\
\hline 4 & 17.5 & 30 & 22.5 & 7.5 \\
\hline
\end{tabular}

In Fig. (5), the variable $H$ is the overall height of the subject. The leg or shank portion is calculated as $0.285 H-0.039 H=0.246 H$

which is measured from the knee center to the ankle center. For the artificial limb, the total height of adjustment adapters connected to the knee joint or ankle joint and the distances from the center of joints to their connected ends can be estimated to be 5 to $10 \mathrm{~cm}$.

Assuming $5 \mathrm{~cm}$ height of adapters and $180 \mathrm{~cm}$ for the subject's height $H$ then the length of the shank $L_{\text {shank }}$ will be

$$
\begin{aligned}
& L_{\text {shank }}=0.246 \mathrm{H}-5 \\
& L_{\text {shank }}=39.28 \mathrm{~cm}
\end{aligned}
$$

Now applying case two in table (1) for example yields that

$H_{\max }=(40+5) / 0.246=182.92 \mathrm{~cm}$

and $H_{\min }=(27.5+5) / 0.246=132.11 \mathrm{~cm}$

which means that the design of case two can fit a subject height of range equals to $(182.92-132.11=50.81 \mathrm{~cm})$. In other words, a range of $12.5 \mathrm{~cm}$ of adjustable shank is approximately 4 times the range of subject height.

Similarly when assuming the height of adapters to be $10 \mathrm{~cm}$, then

$$
L_{\text {shank }}=0.246 \mathrm{H}-10
$$

and for the same design case

$H_{\max }=(40+10) / 0.246=203.25 \mathrm{~cm}$

and $H_{\min }=(27.5+10) / 0.246=152.44 \mathrm{~cm}$

with the same range.

For children amputees, if the relations of anthropometric data were also suitable to apply, then taking case four for example with $5 \mathrm{~cm}$ adapter height yields

$H_{\max }=(30+5) / 0.246=142.27 \mathrm{~cm}$

and $H_{\min }=(22.5+5) / 0.246=111.87 \mathrm{~cm}$ 
with a range of $30.4 \mathrm{~cm}$ of subject height.

Hopefully, this range would provide a good fit for a fairly wide range of patients both adults and children.

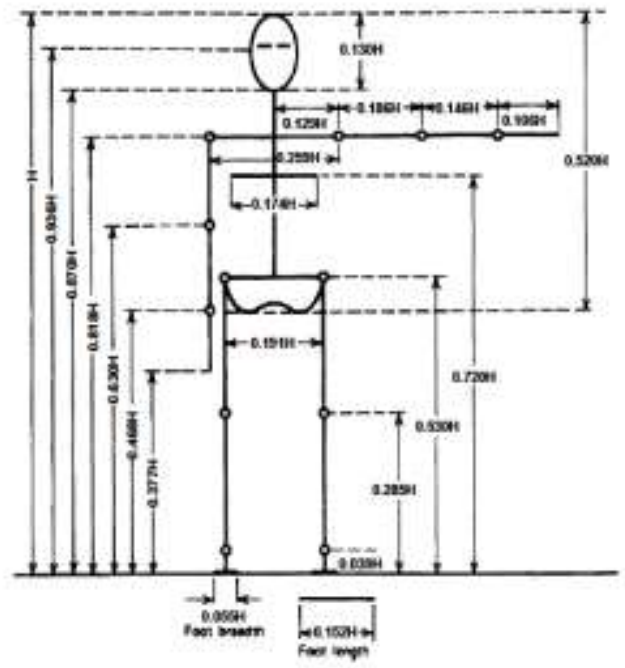

(a)

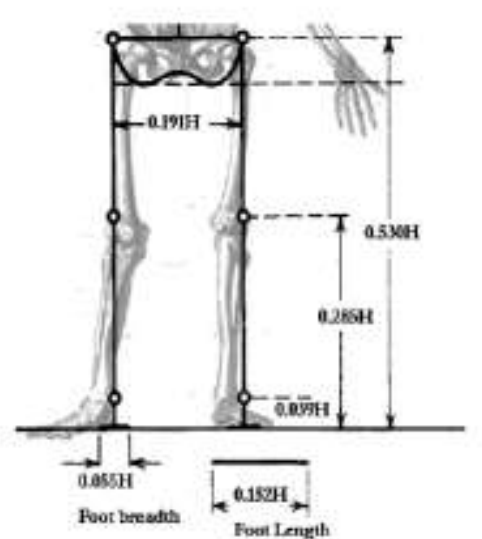

(b)

Fig. (5) Anthropometric data for (a) skeletal system, (b) the lower body [6].

The ankle joint is designed as multi axes joint with two degree of freedom with elastic rubber ring that can be considered as FLEX-Foot (has an energy storing component). The joint permits dorsi-flexion, planter flexion, eversion and inversion. This gives the amputee more stability on bumpy grounds and more natural walking.

Across a functional range of walking speeds, Flex-Foot walking was associated with significantly lower percentages of age-predicted heart rate, lower energy expenditure and improved gait efficiency compared to SACH foot walking [7].

A rubber ring is used to give a spring like action to the ankle and it's placed inside the depression of the foot keel between the keel and the ankle joint. The foot part consists of a wooden foot keel and a thin metal plate attached to the bottom of the wooden keel and a semi-pyramidal shape piece of rubber attached to the back of the keel which serves as a foot heel. The function of the rubber heel is to reduce the impact force on the foot during the heel strike phase if the gait cycle. The metal plate function is to give extra recoil force during the toe off phase of the gait cycle allowing the gait to be more efficient. See fig. (6).

For amputees heavy subjects, modifications to the design have to be done to be on the safe side. (e.g. type of material, thicknesses, diameters ...). For children amputees, the modifications include scaling down the dimensions and using lighter weight materials.

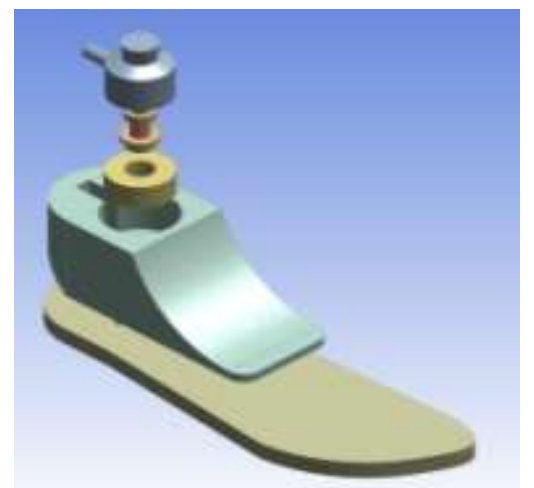

Fig. (6) The parts of ankle joint and foot.

The complete assembled parts of the adjustable lower extremity are shown in fig. (7). 


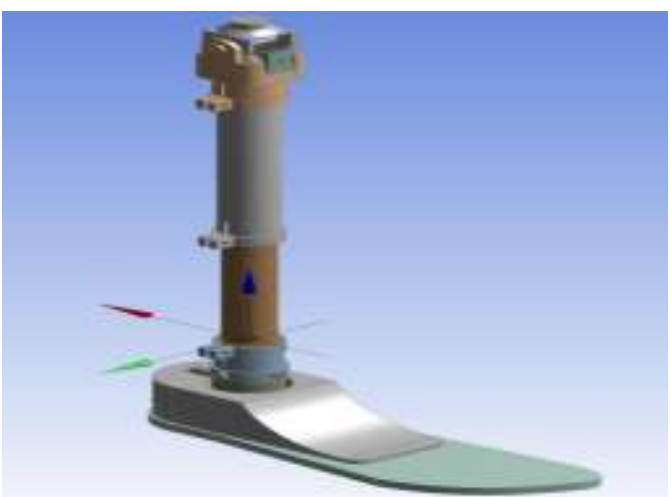

Fig. (7) Complete assembled limb.

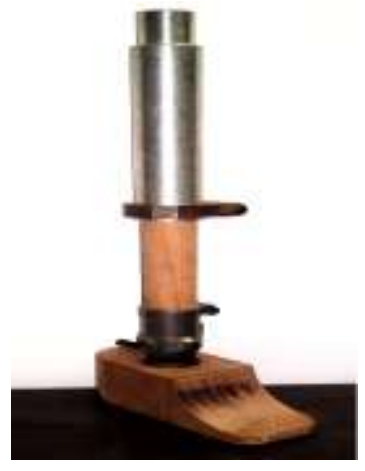

Fig. (8) The manufactured adjustable lower limb.

\section{IMPLEMENTATION OF THE ADJUSTABLE LIMB}

Different types of materials can be utilized to implement the limb. These include: steel, aluminum, titanium, polyvinyl chloride, hard wood, carbon fiber poly ethylene, poly propylene and others. The factors that control the type of material are: yield stress, density, non toxic, corrosion resistance, the maximum load applied, affordability, ability to be molded and machine worked easily. The constructed prototype - shown in fig. (8) - is made from locally found low cost materials that include: aluminum, beech wood, steel, cast iron and rubber. The knee joint is not constructed in this work.

The adjustable shank was made from Aluminum and Beach wood. The foot keel was made from Beach wood. Automobile suspension parts were used in the construction of the ankle joint. These parts are cheap, widely available in the local market, strong and act as a perfect multi-axes joint.

\section{FINITE ELEMENT ANALYSIS}

Finite element method was used to conduct a stress analysis and show the force distribution along the device. The finite element model mesh for knee, shank, ankle and foot are shown in figs. (9) and (10). A fine mesh was used and the meshes have been refined in the high stress areas.
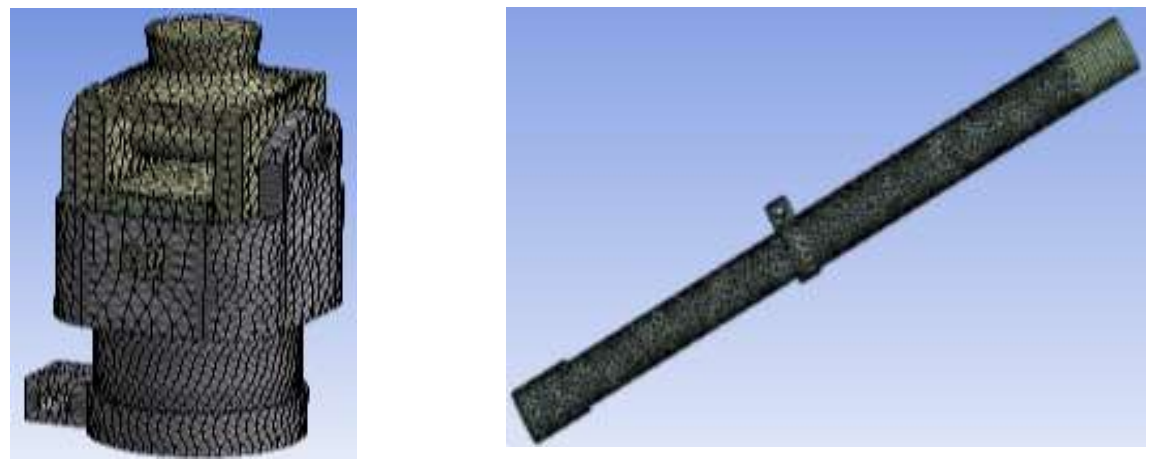

Fig. (9) Meshing of knee joint and shank

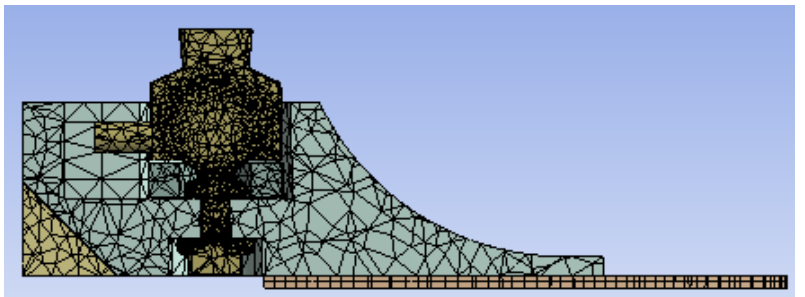

Fig. (10) Section view for the meshing of ankle joint and foot

The prosthetic limb will be analyzed using the international standard for structural testing of lowerlimb prostheses (ISO 10328). This paper focuses on static proof test loading, which forms a design basis for dynamic and cyclic testing. Three test levels are defined in the ISO specification: P3, P4, and P5. P3 testing corresponds to a foot appropriate for a patient with mass of up to $60 \mathrm{~kg}$ (A60), and P4 to a patient with mass of up to $80 \mathrm{~kg}$ (A80). P5, the strongest of the standard strength levels, corresponds to most other patient sizes, including a mass exceeding $100 \mathrm{~kg}$ (A100). 
After finding the locations and directions of loads, the next step is to apply forces and set constraints or supports. Fig. (11) displays the boundary conditions of the knee, shank and ankle-foot models with the forces represented by the red color arrows and the restraints or supports in blue color for the loading condition I and II. Each force was treated separately when solving and finding results

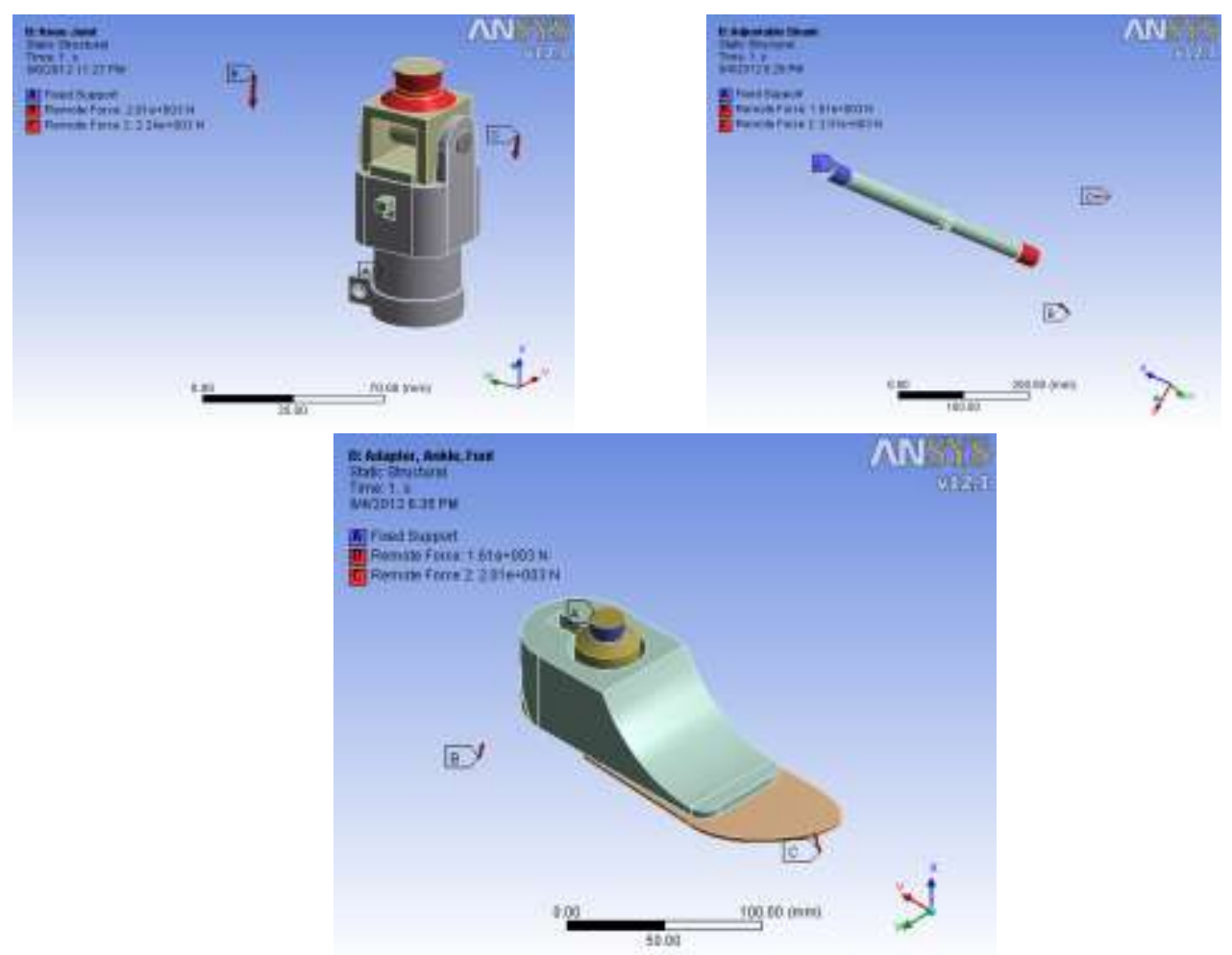

Fig. (11) The boundary conditions of the knee, shank and ankle-foot models

\section{RESULTS AND DISCUSSION}

The results for equivalent (von-Mises) stress, shear stress and total deformation of designed models were obtained by using (ANSYS workbench v12.1) program.

\subsection{Results of Artificial Knee Joint Model}

In fig. (12) for loading condition I, The maximum von Mises and shear stresses occur on the neck of the connection adapter between the upper part of the knee and the point of load application. The amount of these stresses passes the yield limit of the steel used in the analysis. This region lowers the factor of safety of the design. Therefore, two solutions are possible to this problem: either using another type of steel or material with higher yield stress limit, or redesign this area due to its potential failure and instability. The maximum total deformation is on the upper edge of the connection adapter.

In figs. (13 and 14) for loading condition II, The value of von Mises stress and shear stress did not pass the yield limit in this condition. The areas of stress concentration are the neck of the connection adapter on the upper part and the contact area between the cylindrical adapter and body of the lower part of knee. The summary of the results for knee model in loading conditions I and II is shown in Table (2).

Table (2) Summary of the results for knee model in loading conditions I and II

\begin{tabular}{|c|c|c|c|c|c|}
\hline \multicolumn{2}{|c|}{ Loading Condition I (Heal Strike) } & \multicolumn{2}{c|}{ Loading Condition II (Toe Off) } \\
\hline $\begin{array}{c}\text { Max. von } \\
\text { Mises stress } \\
(\mathrm{MPa})\end{array}$ & $\begin{array}{c}\text { Total } \\
\text { Deformation } \\
(\mathrm{mm})\end{array}$ & $\begin{array}{c}\text { Max. Shear } \\
\text { Stress }(\mathrm{MPa})\end{array}$ & $\begin{array}{c}\text { Max. von } \\
\text { Mises stress } \\
(\mathrm{MPa})\end{array}$ & $\begin{array}{c}\text { Total } \\
\text { Deformation } \\
(\mathrm{mm})\end{array}$ & $\begin{array}{c}\text { Max. Shear } \\
\text { Stress }(\mathrm{MPa})\end{array}$ \\
\hline 230.81 & 0.0665 & 128.24 & 184.65 & 0.0807 & 102.76 \\
\hline \multicolumn{4}{|c|}{ The material used is stainless steel with yield stress $=207 \mathrm{MPa}$} \\
\hline
\end{tabular}



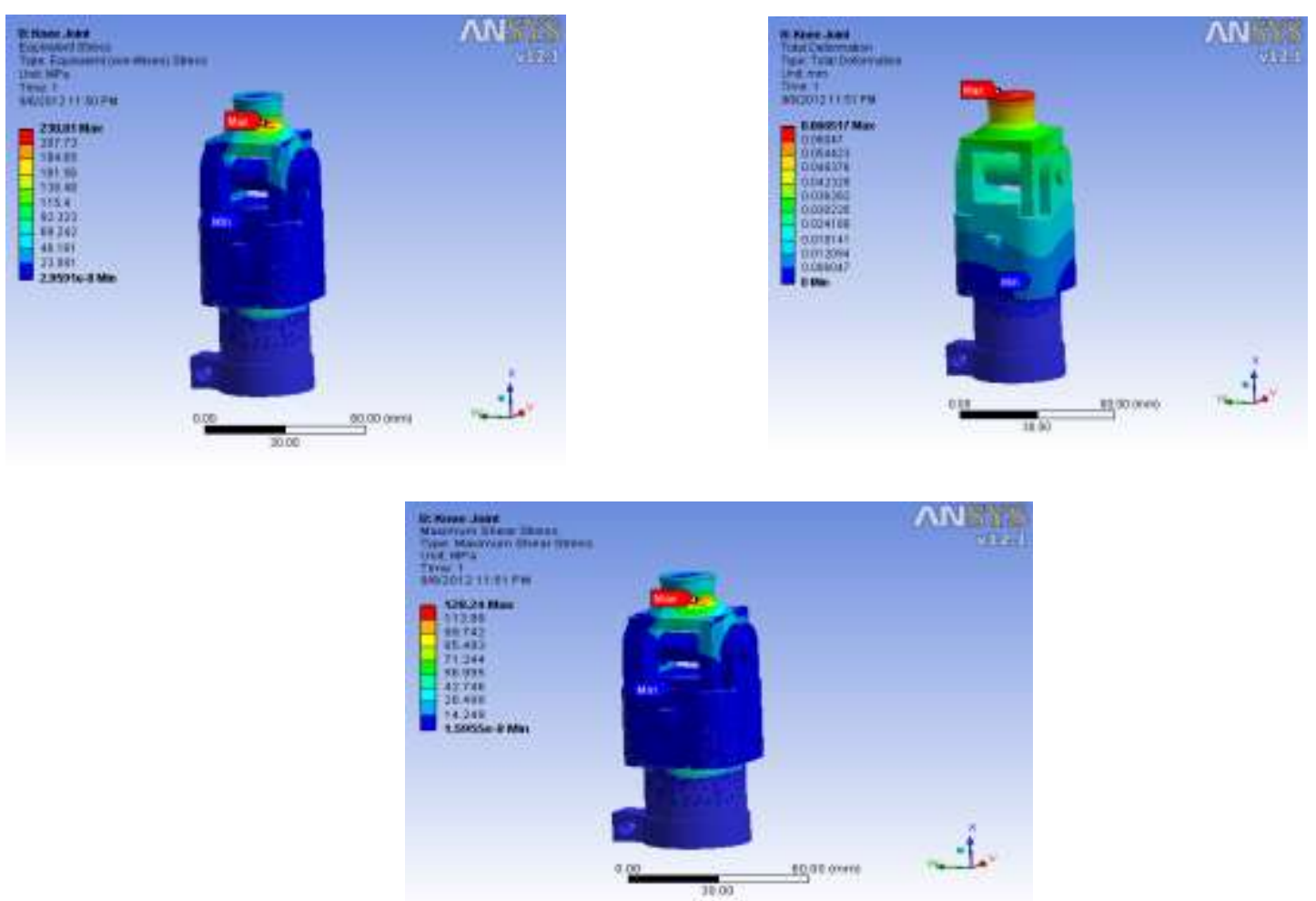

Fig. (12) The equivalent stress, total deformation and shear stress in the in the artificial knee joint stainless steel model for loading condition I (Heel Strike) ISO A100 (2240 N)
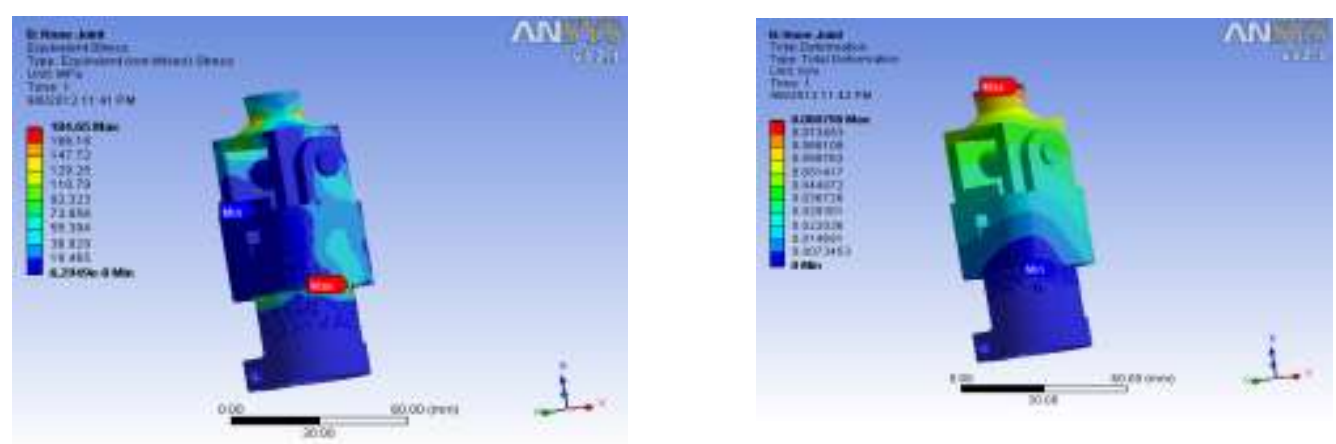

Fig. (13) The equivalent stress and total deformation in the in the artificial knee joint stainless steel model for loading condition II (Toe Off) ISO A100 (2013 N)

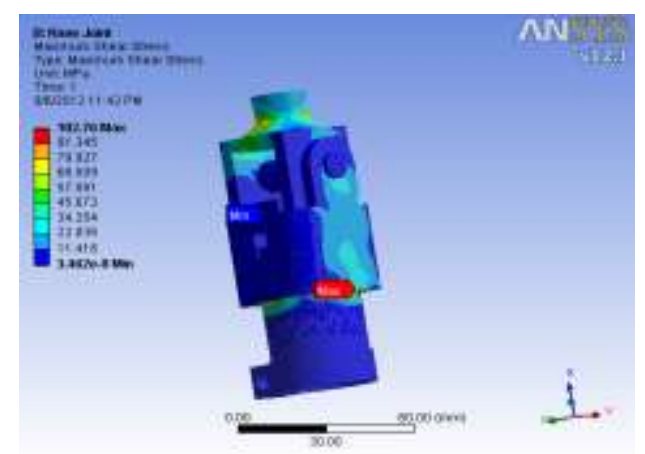

Fig. (14) The shear stress in the in the artificial knee joint stainless steel model for loading condition II (Toe Off) ISO A100 (2013 N)

\subsection{Results of Adjustable Shank Models}

For the adjustable shank models, different types and combination of materials were experimented with. The first material selected was the aluminum alloy, then a combination of aluminum for the upper part and 
beach wood for the lower part of the shank. The aluminum model case was tested for the three levels of testing (which are A100, A80, A60 respectively) according to the ISO standard. The model with beach wood was tested for the A60 level. In figs. (15-19) below, sample results of aluminum A100 and aluminum-wood A60 models for loading condition I and II are shown.

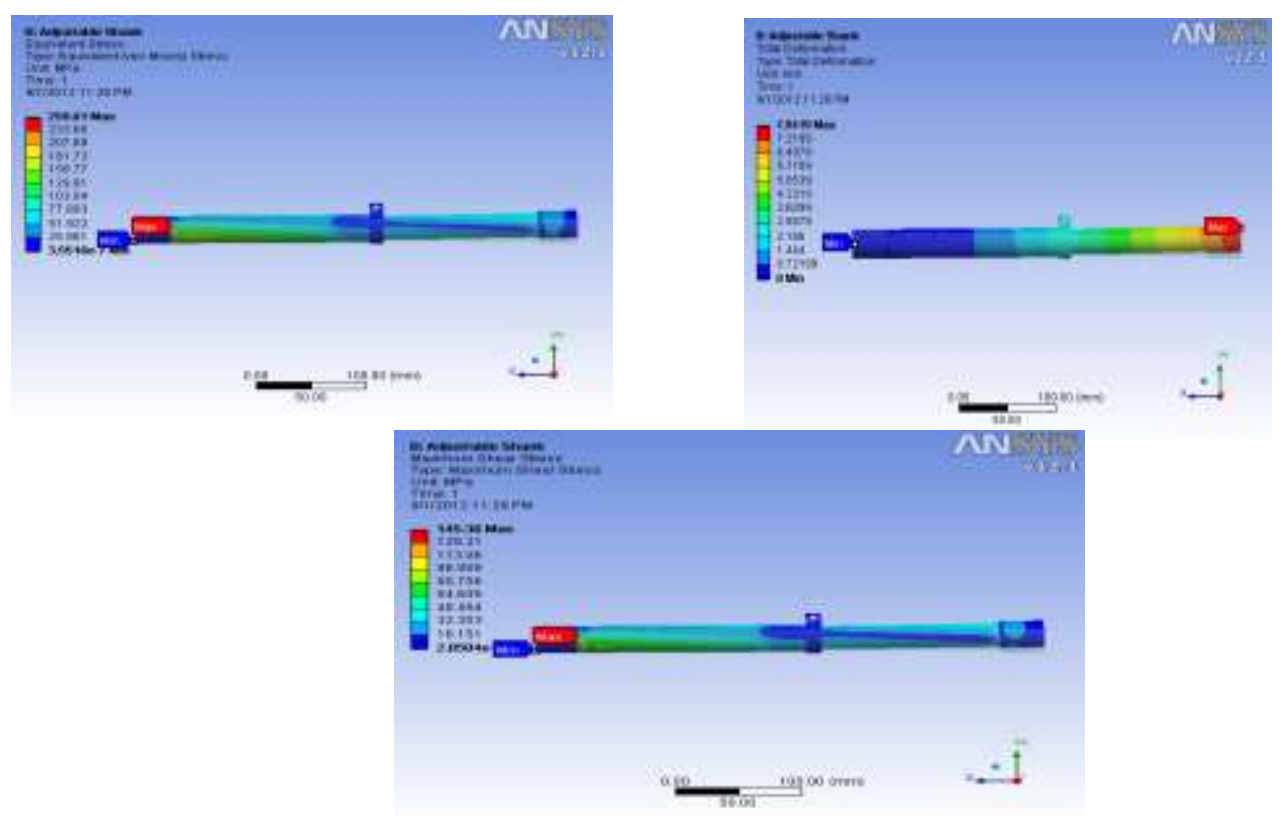

Fig. (15) The equivalent stress, shear stress and total deformation in the adjustable shank Aluminum model for loading condition I (Heel Strike) ISO A100 (2240 N)
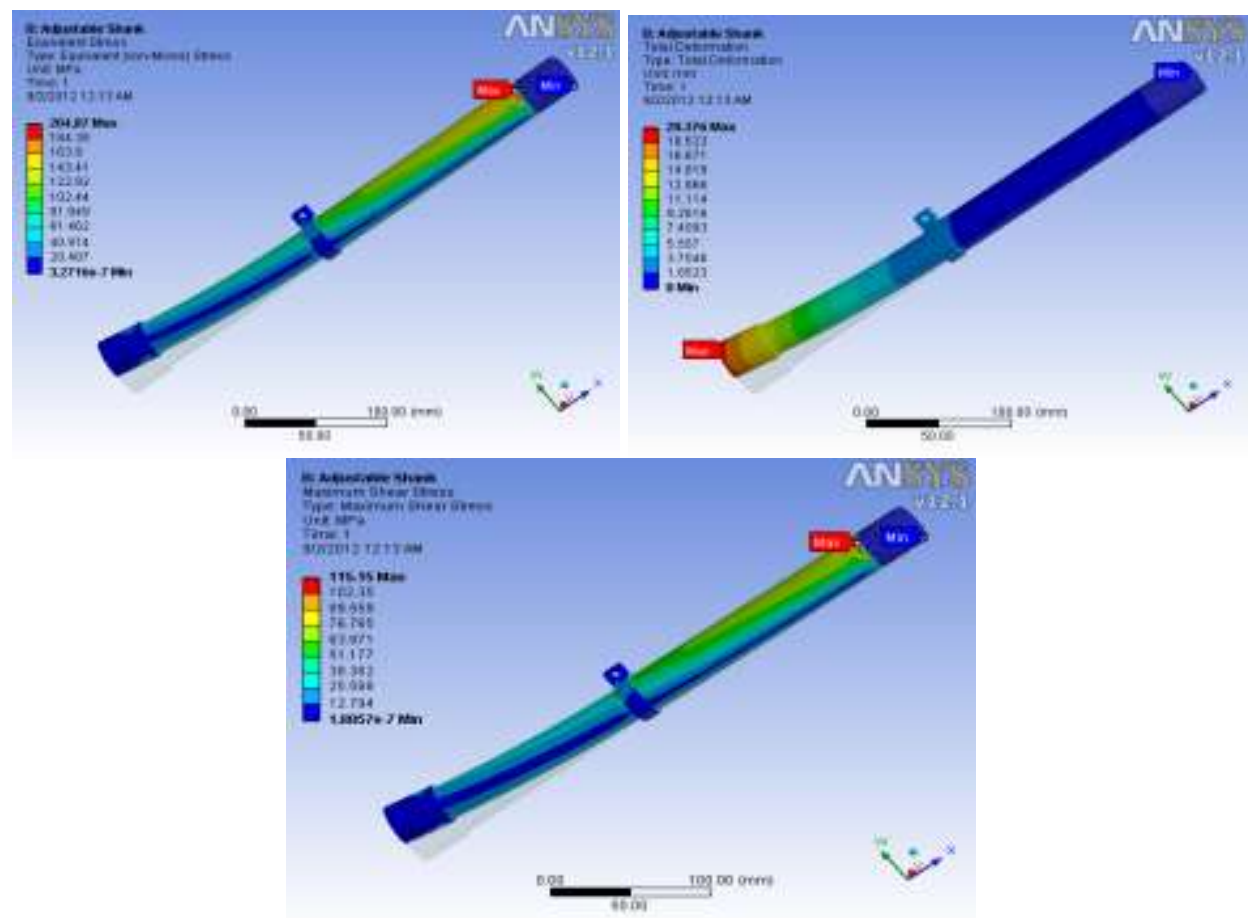

Fig. (16) The equivalent stress, shear stress and total deformation in the adjustable shank (Aluminum-Wooden lower end) model for loading condition I (Heel Strike) ISO A60 (1680 N).

Clearly from figs. (15 and 16), for loading condition I, the maximum von Mises and shear stresses occur at the edge of contact of the upper part with the support. The maximum total deformation occurs at the end of the lower part of the shank.

For the aluminum model with test level A100, The von Mises and shear stresses in test level A100 are just below the yield stress of the aluminum alloy. 
When a combination of aluminum and beach wood is introduced (aluminum for upper part and beach wood for lower part) the results for test level A60 shows that the von Mises stress in the aluminum is ok but for the wood is high and passes the yield stress of the wood in the contact area between the upper and lower parts. The maximum shear stress occurs at the edge of contact of the upper part with the support. The maximum total deformation occurs in the wooden part at the lower end and is far beyond acceptable.
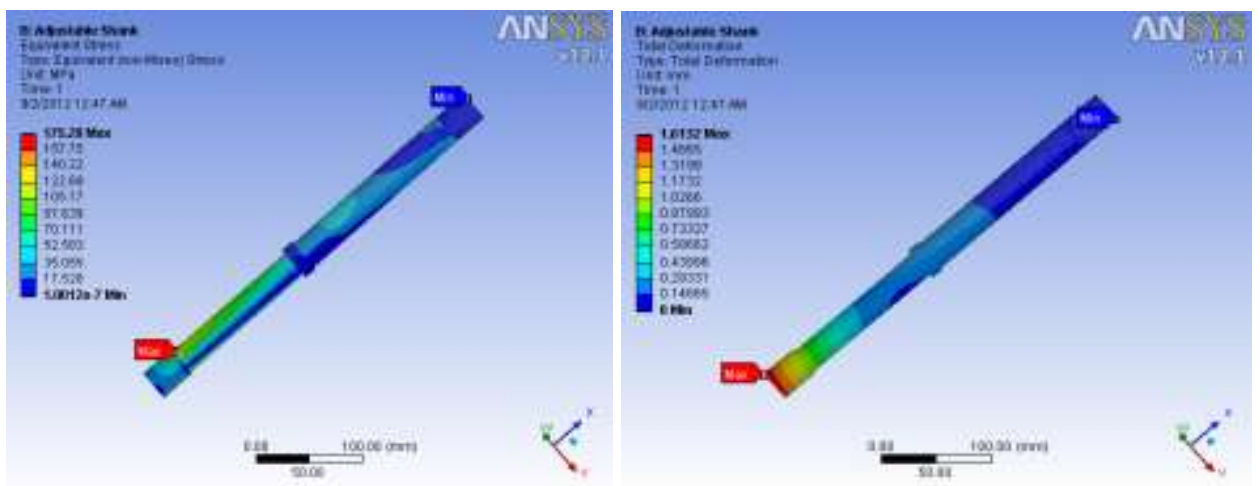

Fig. (17) The equivalent stress and total deformation in the adjustable shank Aluminum model for loading condition II (Toe Off) ISO A100 (2013 N)

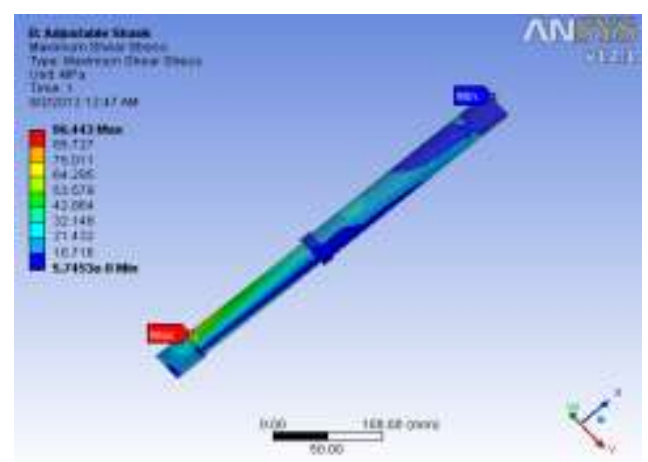

Fig. (18) The shear stress in the adjustable shank Aluminum model for loading condition II (Toe Off) ISO A100 $(2013 \mathrm{~N})$

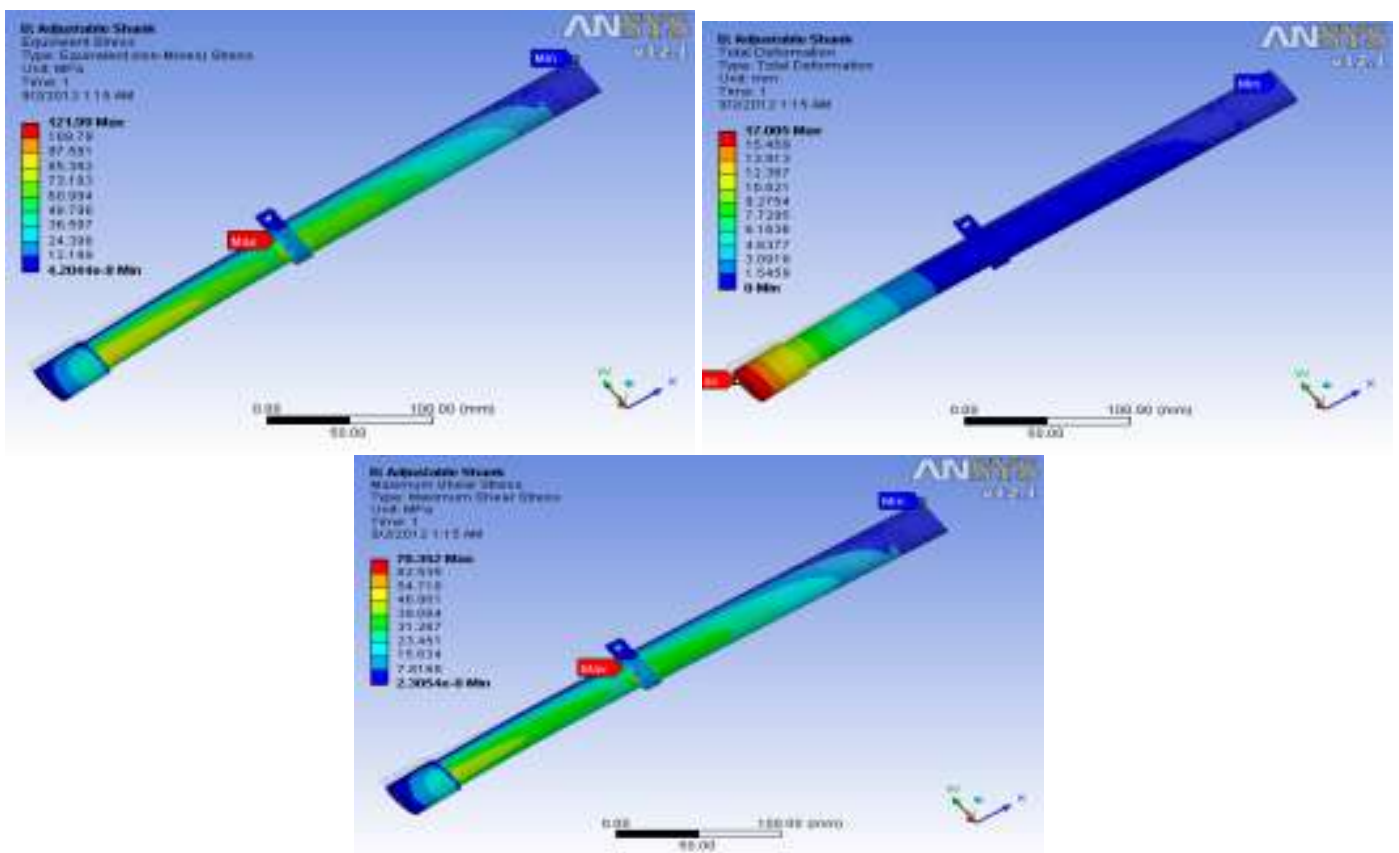

Fig. (19) The equivalent stress, shear stress and total deformation in the adjustable shank (Aluminum-Wooden lower end) model for loading condition II (Toe Off) ISO A60 (1395 N). 
For loading condition II, showed in figs. (17-19), for the aluminum model, the maximum von Mises and shear stresses occur at the beginning of the area of the diameter reduction of the lower part. The maximum total deformation occurs at the end of the lower part of the shank. The von Mises and shear stresses in test level A100 are below the yield stress of the aluminum alloy.

For the model with wooden lower part, the maximum von Mises and shear stresses occur at the edge of contact area between upper and lower parts. The maximum total deformation occurs at the end of the lower part of the shank. The von Mises stress in the aluminum is ok but for the wood is high and passes the yield stress of the wood in the contact area between the upper and lower parts. The maximum shear stress occurs also at the same contact area. The maximum total deformation occurs in the wooden part at the lower end and it is still far beyond acceptable.

The summary of all the results for shank models of the three test levels for loading conditions I and II is shown in Table (3).

Table (3) Summary of all the results for shank models in loading conditions I and II.

\begin{tabular}{|c|c|c|c|c|c|c|}
\hline & $\begin{array}{c}\text { Loading } \\
\text { Condition }\end{array}$ & Type of material & $\begin{array}{c}\text { Test } \\
\text { level }\end{array}$ & $\begin{array}{c}\text { Max. von Mises } \\
\text { stress (MPa) }\end{array}$ & $\begin{array}{c}\text { Total } \\
\text { Deformation } \\
(\mathrm{mm})\end{array}$ & $\begin{array}{c}\text { Max. Shear } \\
\text { Stress (MPa) }\end{array}$ \\
\hline 1 & I & Aluminum & A100 & 259.61 & 7.94 & 145.36 \\
\hline 2 & I & Aluminum & A80 & 237.66 & 7.24 & 133.08 \\
\hline 3 & I & Aluminum & A60 & 181.97 & 5.48 & 101.9 \\
\hline 4 & I & Aluminum-Wood & A60 & 204.87 & 20.37 & 115.15 \\
\hline 5 & II & Aluminum & A100 & 175.28 & 1.61 & 96.44 \\
\hline 6 & II & Aluminum & A80 & 157.13 & 1.41 & 86.45 \\
\hline 7 & II & Aluminum & A60 & 120.32 & 1.03 & 66.18 \\
\hline 8 & II & Aluminum-Wood & A60 & 121.99 & 17 & 70.35 \\
\hline
\end{tabular}

In the design of the adjustable shank, the total length of the shank has great effect on the stresses and deformations produced by the reaction forces and amputee weight. The relation between shank length and the stresses and deformation is approximately linear. See fig. (20).

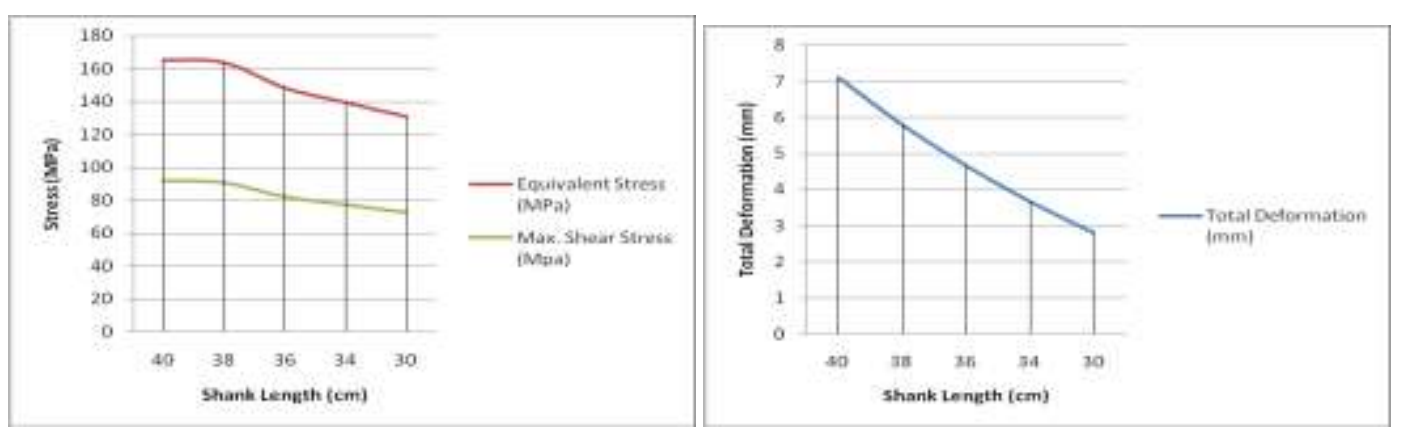

Fig (20) The effect of total shank length on the equivalent stress, shear stress and total deformation.

\subsection{Results of Ankle - Foot Model}

Sample results of the test levels A100 for loading condition I and II for the ankle - foot assembly are shown in figs. (21-23).
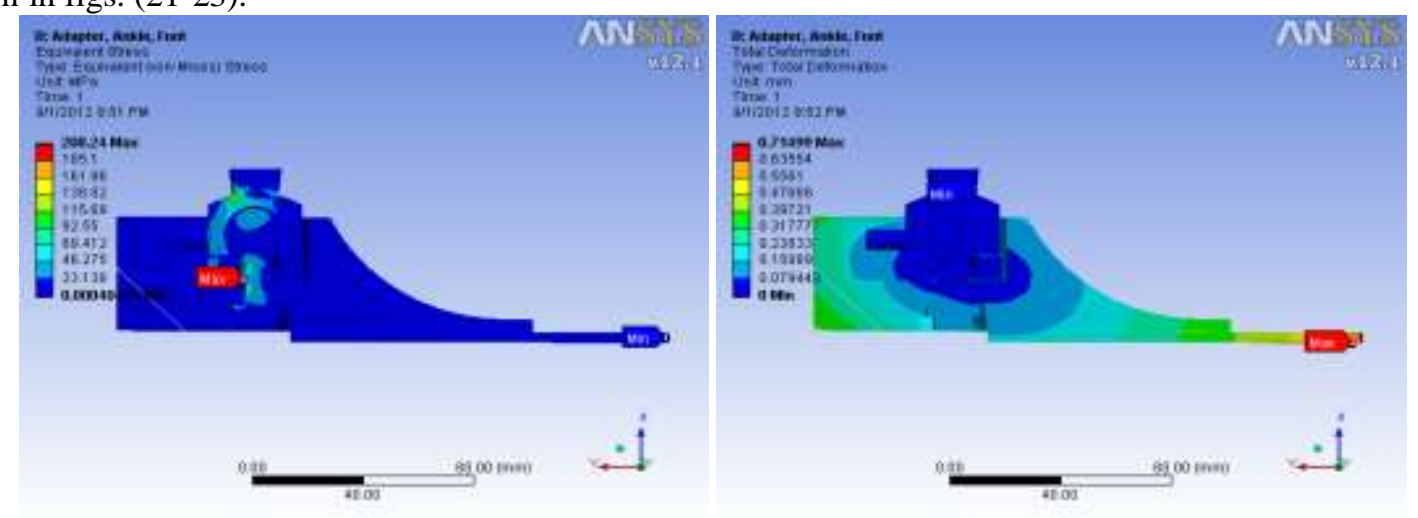


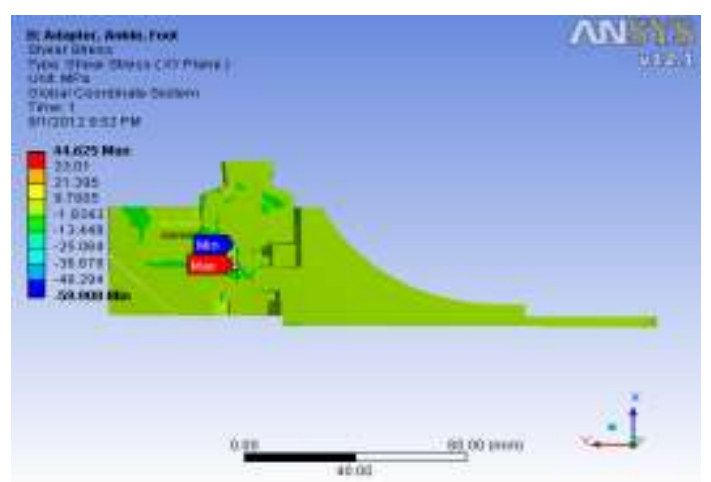

Fig. (21) The equivalent stress, shear stress and total deformation in the ankle-foot model for loading condition I (Heel Strike) ISO A100 (2240 N).

In fig. (21), for loading condition I, The maximum von Mises and shear stresses occur at the shaft of the ankle joint and it is almost equal to the yield stress of the stainless steel for the A100 test level. The maximum total deformation presented in the results occurs at the anterior edge of the foot. This deformation is not a real one that results from the applied loads. The deformation at the heel is the one that should be considered.
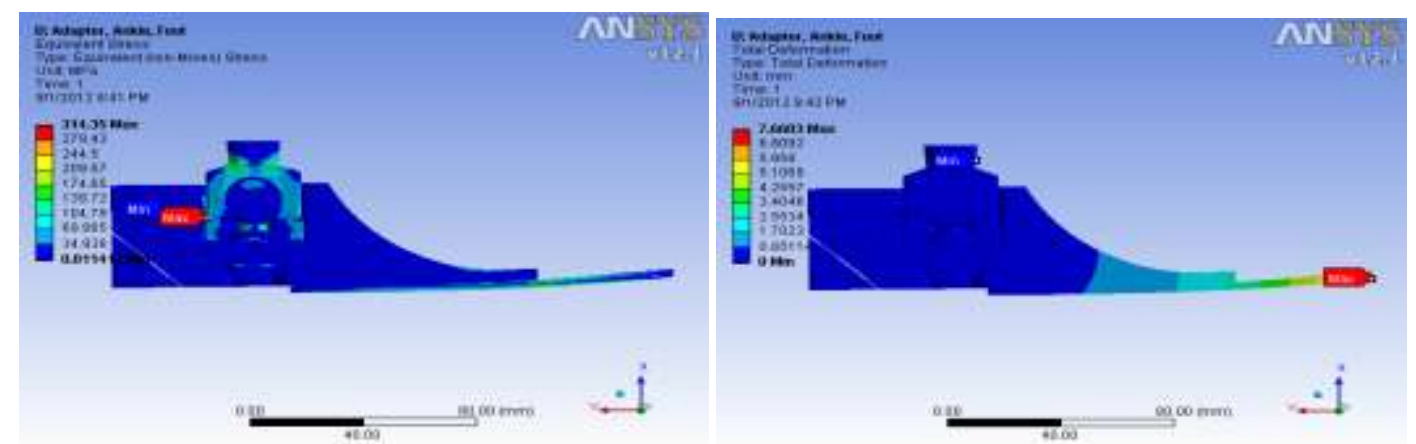

Fig. (22) The equivalent stress and total deformation in the ankle-foot model for loading condition II (Toe-Off) ISO A100 (2013 N).

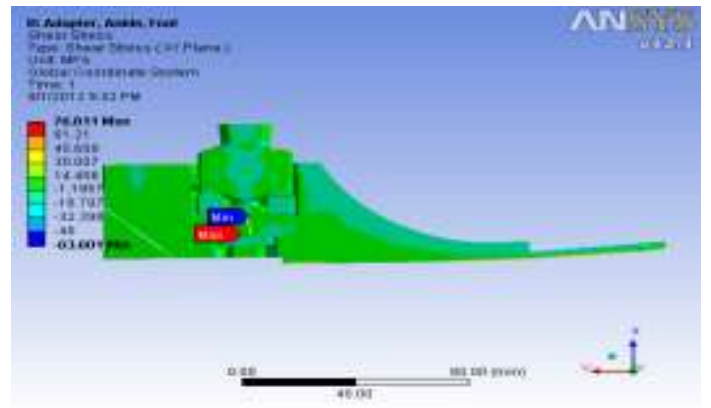

Fig. (23) The shear stress in the ankle-foot model for loading condition II (Toe-Off) ISO A100 (2013 N).

In figs. (22 and 23), for loading condition II, The maximum von Mises stress that is located at the rubber ring is not real because the ring material was assumed as beach wood to force the ANSYS program to reach a solution. The important location that should be considered is the neck of the connection adapter at the top of the ankle joint. The stress in this location is just below the yield stress of the stainless steel in test level A100.

The maximum total deformation presented in the results occurs at the anterior edge of the foot. This deformation is real in this case and results from the applied loads. The maximum shear stress is located at the shaft of the lower part of the ankle joint and its value is within the safe limit.

The summary of all the results for ankle-foot assembly model of the three test levels for loading conditions I and II is shown in Table (4). 
Table (4) Summary of all the results for ankle joint and foot model in loading conditions I and II.

\begin{tabular}{|c|c|c|c|c|c|c|}
\hline & $\begin{array}{l}\text { Loading } \\
\text { Condition }\end{array}$ & $\begin{array}{l}\text { Type of } \\
\text { material }\end{array}$ & Test level & $\begin{array}{c}\text { Max. von Mises } \\
\text { stress (MPa) }\end{array}$ & $\begin{array}{c}\text { Total } \\
\text { Deformation } \\
(\mathrm{mm})\end{array}$ & $\begin{array}{l}\text { Max. Shear } \\
\text { Stress (MPa) }\end{array}$ \\
\hline 1 & $\mathrm{I}$ & \multirow{6}{*}{$\begin{array}{l}\text { Stainless steel, } \\
\text { Beach Wood }\end{array}$} & A100 & 208.24 & 0.71 & 44.62 \\
\hline 2 & I & & A80 & 191.97 & 0.65 & 41.13 \\
\hline 3 & $\mathrm{I}$ & & A60 & 149.67 & 0.51 & 32.07 \\
\hline 4 & II & & A100 & 314.35 & 7.66 & 76.81 \\
\hline 5 & II & & A80 & 282.81 & 6.89 & 69.10 \\
\hline 6 & II & & A60 & 217.84 & 5.30 & 53.23 \\
\hline
\end{tabular}

VII.

\section{CONCLUSIONS}

Adjustable artificial lower limb is a good and easy solution to be used by amputee patients that who need to change the length of pylon easily without having to replace their artificial limbs. Patient who need to wear different pair of shoes can now easily do that. Children amputees who grow in length rapidly will use the same limb for longer times and cut-off the extra expenses and visits to the prosthetic workshops. Patients who need to change their feet type can know do that without the need to by two sets of artificial lower limbs.

Based on the results and the discussion, the beach wood is cheap, light in weight, widely available but it has poor mechanical properties that make it only useful to be used for children with lower weights. Stainless Steel has good mechanical properties to be used in the manufacturing of the parts of the artificial limbs. But its relatively high weight contributes to the overall discomfort of the patient. On the other hand, the aluminum proofs to be the material of choice for making artificial limbs. Aluminum has very good strength to weight ratio, affordable, non-corrosive and easy to work with.

Certain parts of the artificial limb develop stresses that are higher than the yield stress of the aluminum and some constantly moving parts need higher wear and tear resistance the aluminum, these parts (like the knee joint) should be made of stronger materials like carbon-steel.

In final word the designer should not make final recommendations for his designs and the types of materials without checking that the stresses are lower than the yield limit under the ISO Test Standard Loading Conditions. Then the model is safe and appropriate for use in the field.

\section{REFERENCES}

[1] Mark R. Pitkin, Biomechanics of Lower Limb Prosthetics (Springer-Verlag Berlin Heidelberg, 2010).

[2] Erin Strait Prosthetic Resident, Prosthetics in Developing Countries (January 2006).

[3] Prasanna K Lenka, Amit R Chowdhury, Ratnesh Kumar, Design \& Development of Lower Extremity Paediatric Prosthesis, a Requirement in Developing Countries, IJPMR, Kolkata, 2008 April.

[4] Bauer J. J., Defining Intensity of Skeletal Loading in Children, doctoral diss., Oregon State University, 2006.

[5] Susan J. Hall, Basic Biomechanics, third edition (McGraw-Hill, 1999, ISBN 0-07-116373-5).

[6] Rose J., Gamble J.G., Human Walking, 3rd ed. (Philadelphia: Lippincott Williams \& Wilkins, 2005).

[7] Pamela A. Macfarlane, David H. Nielsen, Donald G. Shurr, Mechanical Gait Analysis of Transfemoral Amputees: SACH Foot Versus the Flex-Foot, Journal of Prosthetics and Orthotics/Volume 9, Number 4/Fall 1997 esign. 